\title{
Prendre la décision de fraternité
}

\section{Agnès Perrin-Doucey}

\section{OpenEdition}

Journals

Electronic version

URL: http://journals.openedition.org/trema/5721

DOI: $10.4000 /$ trema. 5721

ISSN: 2107-0997

\section{Publisher}

Faculté d'Éducation de l'université de Montpellier

\section{Electronic reference}

Agnès Perrin-Doucey, « Prendre la décision de fraternité », Tréma [Online], 53 | 2020, Online since 01 March 2020, connection on 09 March 2020. URL : http://journals.openedition.org/trema/5721 ; DOI https://doi.org/10.4000/trema.5721

This text was automatically generated on 9 March 2020

Trema 


\title{
Prendre la décision de fraternité
}

\author{
Agnès Perrin-Doucey
}

\section{EDITOR'S NOTE}

Dans l'ensemble de notre texte, nous utiliserons le mot fraternité sans lui adjoindre sa déclinaison féminine de sororité, nous appuyant sur l'analyse de ces deux termes proposée par C. Chalier (2003, p. 126-133).

à Xavier,

Il n'y a pas d'éducation sans valeurs. Même si l'on réduit l'éducation à l'enseignement scolaire, on apprend à l'école. Or qu'est-ce qu'apprendre sinon passer d'un état à un autre, plus souhaitable? Apprendre, c'est se délivrer d'une ignorance, d'une incertitude, d'une maladresse, d'une incompétence, d'un aveuglement, c'est parvenir à mieux faire, à mieux comprendre, à mieux être. Or, qui dit «mieux » dit valeur (Reboul, 1992, p. 1).

\section{Contexte et visées}

\section{Contexte}

\section{Penser la fraternité au cœur de la société}

1 En février 2015, un mois après le violent séisme citoyen provoqué par les attentats terroristes islamistes contre le magazine satirique Charlie-Hebdo, une jeune policière à Montrouge, et un hypermarché Casher situé Porte de Vincennes, le philosophe Abdennour Bidar publie un Plaidoyer pour la fraternité. Dans cette œuvre, conçue comme 
« une longue lettre » adressée à ses « frères et sœurs humains de tous bords, de toutes origines ", il enjoint ses contemporains à prendre en compte «l'urgence d'œuvrer tous ensemble maintenant à quelque chose de très simple, de très beau et de très difficile : la fraternité. La fraternité tout court, et pas seulement la fraternité de tel sang ou de telle religion. » (2015, p. 11). Considérant l'absence de fraternité dans le vivre-ensemble de la société contemporaine, ou sa rareté, comme étant ce qui fait le plus souffrir, il propose alors que :

Cet idéal devienne maintenant une véritable direction, un projet de société concret, un projet de civilisation vers lequel nous ferons converger d'abord nos cœurs, et à partir d'eux nos éducations, nos institutions, nos engagements, nos métiers et nos forces vives. (p. 11-12)

2 Pour Patrice Viveret, « lorsque le tragique revient, lorsque l'enjeu est la vie et la mort, tout le reste passe au second plan et peut paraitre franchement dérisoire» $(2015$, p. 23). Dès lors, il affirme lui aussi la nécessité de réengager l'essentiel, qui «se trouve dans l'exigence de redonner tout son sens à l'esprit de fraternité » (p. 25). Comme A. Bidar, il considère que « le devenir de notre famille humaine, sa perte ou son salut, se jouera sur l'aptitude de notre fratrie à enrichir son art d'aimer et son humanité, pour construire une humanité plus conviviale en développant [...] l'intérêt pour soi et l'intérêt pour autrui » (Ibid., p. 70).

3 Dès lors, le lien de fraternité, bien souvent dissocié de celui de citoyenneté, semble être à même de se régénérer dans cette période troublée, précisément parce que l'humain se trouve violemment confronté à l'inhumaine barbarie dont l'homme sait être capable (Chalier, 2003).

\section{Penser la fraternité au cœur de l'école}

4 Et c'est peut-être aussi, parce qu'il lui semble nécessaire de ne pas perdre de vue que la formation du citoyen se construit parfois par les épisodes tragiques qu'il traverse, que le Ministère de l'Education Nationale ${ }^{1}$ engage à cette époque une grande mobilisation nationale de l'école pour les Valeurs de la République (2015a). Il publie, dans l'urgence d'une réponse politique attendue voire réclamée par des citoyens indignés et/ou désorientés, onze mesures nécessaires pour former les élèves français au pacte citoyen, donc à la décision de fraternité en tant qu'un des piliers fondateurs des valeurs républicaines, comme l'affirme la devise. Pour Najat Vallaud Belkacem, alors ministre de l'éducation nationale, si l'école « assume pleinement son rôle éducatif » ne tolérant "aucune remise en cause des valeurs de la République", elle n'est cependant pas " étanche aux dérives et aux troubles de notre société » (2015a, en ligne). Elle affirme à ce propos que l'institution doit aussi pouvoir réfléchir à ce que les prises de position, ou les doutes exprimés par certains élèves au moment des hommages rendus par la nation aux victimes des attentats ${ }^{2}$, révèlent de la fracture avec les valeurs citoyennes qui devraient fonder notre commun partageable. Rappelant les difficultés récurrentes à lutter contre les déterminismes sociaux, elle ajoute enfin qu'il faut mettre en œuvre une politique active de mixité sociale en agissant notamment sur les collèges. Dans cette perspective, elle lit la rupture constatée entre l'école, ses valeurs, et une partie de ses élèves à l'aune de la fracture qui gangrène le pays depuis plusieurs décennies. De fait, les onze mesures publiées invitent à "mettre la laïcité et la transmission des valeurs républicaines au cœur de la mobilisation de l'École», et à "développer la citoyenneté et la culture de l'engagement avec tous les partenaires de l'École ». Enfin, 
ces mesures doivent aussi « combattre les inégalités et favoriser la mixité sociale pour renforcer le sentiment d'appartenance dans la République " (2015a, en ligne). Ainsi, le gouvernement de l'époque semble acter l'idée que sans justice sociale, point de construction commune possible et que sans construction commune, point de citoyenneté possible.

5 L'institution invite enfin l'Université à participer à cette grande mobilisation nationale. De fait, elle considère comme nécessaire l'élaboration d'une pensée critique à caractère philosophique, politique, social, culturel mais aussi didactique et pédagogique. En effet, un tel projet implique de penser scientifiquement les modalités de cet effort d'une «transmission » centrée sur des valeurs, dans un contexte institutionnel qui renforce la dimension pragmatique de la formation morale et civique (MEN, 2015b).

\section{Visées}

\section{Penser la complexité}

6 Cependant pour Edgar Morin (2019), observateur et analyste des crises sociales contemporaines, si la fraternité doit effectivement permettre de résister à la cruauté du monde, «elle ne peut venir d'une injonction étatique supérieure, elle doit venir de nous » (p. 15). Il remet alors en cause la pertinence pragmatique de ce présupposé de la transmission déterminé par les programmes. En effet, l'adhésion au concept de fraternité, plus peut-être encore que l'adhésion aux deux autres piliers du triptyque, pose véritablement problème s'il reste inhérent à nos choix personnels, alors même qu'elle fonde l'équilibre de notre agir citoyen (Ibid., p. 21). Il apparait bien là, comme à chaque fois que l'on interroge une éducation aux valeurs nécessaires pour construire du commun à partir du divers, une difficulté profonde, un paradoxe puissant, un défi majeur. Difficulté, paradoxe et défi, qui pour se résoudre, nécessitent soit de reconsidérer le projet de société en refusant l'idée de valeurs communes partagées; soit de se demander comment la société peut agir pour inciter, décider, porter la majorité des citoyens ou futurs citoyens vers cet engagement civique. Cette seconde perspective implique d'assumer une rupture nécessaire avec l'« excès de Fraternités minuscules de type communautaire, et [le] déficit de Fraternité majuscule intégrant les premières » (Debray, 2009, p. 310). C'est ce défi que l'école doit donc relever, puisqu'elle participe fortement, mais pas exclusivement, à la construction du citoyen de demain.

\section{Dépasser le contexte premier}

7 C'est précisément dans ce contexte politique et philosophique posttraumatique ayant impliqué au moins ponctuellement un sursaut de « Fraternité majuscule » qu'est né, en 2016, un projet de colloque ${ }^{3}$. L'écriture de cet ouvrage est mue, quant à elle, par le désir de se distancier du tragique, pour construire une pensée collective, plurielle conçue dans une perspective polydisciplinaire ${ }^{4}$. Nous souhaitons tresser des relations entre éducation et fraternité, tressage qui, selon nous, s'avère indispensable au tissage solide, voire durable, d'un commun partageable. En 2020, la résonance et la nécessité de ce travail apparaissent plus clairement encore : la fracture sociale et citoyenne, loin de se résoudre, s'accroit. Avec elle se renforce le cortège des violences, des outrances, des extrémismes puissants et souvent contradictoires qui apparaissent ou réapparaissent dans les périodes troublées. Le commun partageable devient alors invisible et inaudible. 
Il semble se diluer dans un individualisme forcené, trop souvent aveuglé par la haine d'une altérité, quelle qu'elle soit, considérée avec l'effroi irrépressible qui surgit, pour certains, quand l'inconnu fait face. Ce sont précisément ces questions que nous voulons aborder ici. Nous nous demandons finalement comment l'éducation à l'école pourrait favoriser l'appropriation de la fraternité, principe construit sur les valeurs humanistes. Nous pensons en effet, à l'appui des travaux d'E. Morin, qu'il n'est pas envisageable de transmettre la fraternité (donc de l'enseigner) et qu'il s'agit plutôt de convaincre les élèves de la nécessité citoyenne d'un engagement personnel pour l'autre.

\section{Construire le projet}

Par pragmatisme, par souci de l'évidente cohérence qu'implique l'écriture scientifique, il est indispensable d'introduire notre propos par une réflexion à vocation épistémologique. Dans cette perspective, en premier lieu, nous avons à cerner les acceptions du concept de fraternité, dans ses dimensions «minuscules de type communautaire » tout comme dans sa vocation « majuscule » (Ibid.), ou « métapolique » (Chalier, p. 151). Ainsi, nous tentons de dégager un sens - tout à la fois signifiant et directionnel - au cœur même de sa polysémie. Dans un second temps, une fois cette clarification posée, nous chercherons à mettre en évidence la place dans le système éducatif de la fraternité préalablement définie comme une valeur-principe (Heinich, 2017). Enfin nous proposerons quelques-unes des conditions nécessaires à une appropriation possible d'un concept que nous considérons d'emblée comme réellement partageable par les élèves.

Ainsi, espérons-nous développer leur capacité à s'engager, pour les inciter à prendre, un jour peut-être, une décision de fraternité dont les enjeux et les modalités restent à définir. Ainsi, espérons-nous aussi participer, de fait, avec toute la modestie requise tant l'œuvre est vaste, à la construction d'une société plus juste et plus solidaire.

\section{Des fraternités}

\section{Étymologie}

10 Le terme fraternité recouvre plusieurs acceptions dont le point commun reste l'idée de lien, de relation - plutôt positive - entretenu par au moins deux personnes. C'est dans la spécification du rapport créé, ou qui se crée entre ces êtres que les définitions diffèrent et évoluent.

11 L'étymologie renvoie au latin fraternitas dont la racine est frater (frère). Il évoque donc la relation entre frères au sens biologique du terme. Malgré cet étymon, le terme latin ne s'attache pas spécifiquement et uniquement à la notion de parentalité. En effet, Gaffiot (1934, p. 685) atteste l'existence de trois signifiés différents que les auteurs lient alternativement à la parentalité (Lactance), mais aussi et antérieurement à la relation de confraternité entre les peuples (Tacite) et enfin entre chrétiens (selon la version de la Bible).

Le lien fraternel trouve donc son origine dans une dimension génétique, qui, quelle que soit la relation sociale et émotionnelle unissant les frères et sœurs, reste inaliénable. Ici, l'idée de fraternité participe à une définition identitaire de l'individu, établissant son origine, reconnaissant son patrimoine. Elle atteste son appartenance à une famille 
spécifique du moins génétiquement, y compris quand les individus ne se connaissent ou ne se reconnaissent pas comme tels. Par extension, ce lien biologique peut être détourné par la mise en œuvre d'une construction sociale impliquant une restructuration familiale. De lien génétique, il devient un lien choisi (au moins par les parents qui le construisent), reconnu juridiquement, allant jusqu'à rendre légale ou légitime une appartenance identitaire. Il redéfinit symboliquement l'origine par un caractère familial non biologique. Ainsi fonctionne l'adoption plénière, voire simple : elle légalise une situation qui s'établit au sein d'une famille par la volonté des parents et l'accord des institutions en charge des aspects juridiques de ce projet. La fraternité est donc d'abord identitaire aux doubles sens biologique et juridique. Elle engage un entre-soi permettant d'établir une identité spécifique ${ }^{5}$ au cœur de l'altérité. Cette fraternité originelle n'implique pas nécessairement l'affection mutuelle. Pourtant, dans sa conception-même, elle initie dans la relation, un mouvement puissant des affects positifs comme négatifs. Les récits mythologiques mettant des frères ${ }^{6}$ en scène le montrent; nous les explorerons dans un paragraphe ultérieur.

\section{La polysémie des fraternités}

\section{Des fraternités closes}

13 La polysémie du vocable se dévoile essentiellement à la faveur de glissements métaphoriques. Ils sont utilisés pour comparer des formes relationnelles, au moins symboliquement, à celles reliant les membres d'une fratrie génétique. Ces liens sociaux et culturels, qui se proclament fraternels, s'établissent à partir d'une ou plusieurs propriétés communes sur lesquelles s'élaborent les relations. Elles permettent aux individus de se reconnaitre partiellement dans les choix ou les actions de l'autre. Parfois, la métaphore est filée jusqu'au point que deux êtres souhaitent ou décident de mêler leur sang, symboliquement ou réellement, en signe d'un engagement indissoluble. Il s'agit alors de construire un lien horizontal sur les fondements de la verticalité originelle.

Dans tous ces cas, qu'elle soit issue de la fratrie, du désir de simuler par un acte symbolique son essence biologique, ou de la confraternité, la fraternité établie favorise le développement d'une communauté d'appartenances. Elles constituent les caractéristiques spécifiques des composantes identitaires qui s'adjoignent même si la nature de celles-ci différent. Elles sont, selon les cas, biologique, sentimentale, sociale, culturelle, professionnelle, idéologique, religieuse, (etc.). Ces appartenances reliées par des dimensions génétiques et culturelles forment des micro-identités collectives pluricommunautaires. Elles constituent la somme des « fraternités minuscules de type communautaire » évoquée par R. Debray. Dans la plupart de ces cas, cette recherche d'une relation fraternelle ne semble pas déboucher sur la construction d'une ouverture des mêmes vers les autres, ceux qui ne sont pas mêmes. En effet, penser leur altérité implique d'observer une différenciation problématique pour l'équilibre fraternel préalablement établi.

\section{Vers une fraternité ouverte}

Les projets d'A. Bidar, de P. Viveret ou de R. Debray ne se situent pas dans cette définition, qu'ils écartent par des précautions oratoires liminaires. Ils envisagent donc une acception plus universelle à l'idée de fraternité. Loin d'opposer les êtres du fait de 
leurs différences, cette conception nouvelle pourrait les rassembler. Sans refuser les différences et appartenances identitaires, elle s'institue sur une ambition commune ancrée dans le tréfonds de leur humanité. Ce concept de "Fraternité majuscule » à vocation universelle s'élabore donc à partir de l'idée que les hommes sont semblables avant d'être autres. Cependant, il ne se donne pas pour mission de dissoudre les composantes de leur altérité dans une uniformisation nécessairement castratrice pour l'individualité. Dès lors, la dimension englobante inscrite dans un principe d'universalité ne peut s'élaborer en faisant table-rase des appartenances inhérentes aux individualités.

\section{Construire les réseaux de sens}

Pour E. Morin, la construction humaine et sociale passe effectivement par la construction du rapport de soi à l'autre. Expliquant que l'homme est doté du « logiciel égocentrique du moi-je » (2019, p. 25), il rappelle qu'il a aussi besoin, dès l'enfance, « du "nous" et du "tu" qui reconnait "toi" comme sujet analogue à "soi" et proche affectivement de soi, tout en étant autre. » (Ibid., p. 26) Il considère que « les sources du sentiment qui nous portent vers autrui, collectivement (nous) ou personnellement (tu), sont les sources de la fraternité.» (p. 29). Jacques Attali (1999) rejoint partiellement au moins cette acception qui met le sentiment au centre du processus de fraternité. Pour lui, la fraternité doit être pensée au regard des utopies ayant traversé l'édification des civilisations. Elle se définit nécessairement comme un concept complexe et en mouvement. Ne posant pas comme un préalable l'origine génétique ou culturelle du lien, il interroge plutôt la nature des affects qui composent la relation humaine.

Dans son énoncé le plus simple, tiré du sens commun, on peut la définir comme l'attitude par laquelle chacun s'engage à ne pas nuire à autrui [...]. Si on limitait là sa définition, elle ne serait qu'une utopie négative, une protection contre la violence; elle ne constituerait pas une espérance de bonheur ou d'épanouissement. En allant un peu plus loin et en s'en tenant encore à ce que l'étymologie annonce, on peut la définir comme un ordre social dans lequel chacun aimerait l'autre comme son propre frère [...]. (Attali, 1999 : 1214).

En cela, les deux chercheurs s'opposent aux " partisans du réalisme, voire du cynisme et de l'inespoir, [qui soulignent] la fragilité de tous les liens humains, dans la banalité de la vie quotidienne comme lors des tragédies accablantes et terribles » (Chalier, 2003, p.8). Ils invitent à se demander «Pourquoi le lien fraternel aurait-il le moindre privilège? » (Ibid.)

18 La réponse se trouve, pour ces deux chercheurs, dans une analyse épistémique des mythes fondateurs.

[...] Mais il faut se méfier de l'étymologie : aimer les autres comme on aime son frère ne constitue en rien une garantie d'amour. Car tous les mythes nous le crient : l'amour ne caractérise nullement la relation naturelle entre membres d'une même famille, et surtout pas entre frères. Au contraire, entre ces derniers, c'est plutôt la haine qui est de règle. (Attali, 1216). 


\section{Violence et fraternité}

\section{Le fratricide au cœur de la mythologie}

19 Violence et fraternité ont partie liée, comme deux polarités qui ne peuvent advenir l'une sans l'autre. Pour C. Chalier, cette forme fraternelle, qui se conçoit comme «close » (Morin, p. 30) se définit aussi par «la force d'un lien construit sur une réalité partagée [...] que l'on espère maintenir vivante ou dont on désire retrouver la signification [...] » (p. 9). Dès lors, elle s'interroge sur ce « qui fonde la fraternité et sur ce qui entretient la vivacité de sa pensée même lorsque rien ou presque rien, dans la réalité, ne semble la corroborer. » (Ibid.)

Les récits mythologiques, notamment la « source biblique et la source grecque » (Ibid.) ont tissé la trame de cette culture occidentale " portant un regard exempt de naïveté ou de bons sentiments» (Ibid.). J. Attali en fait le fondement de son argumentaire. Beaucoup de civilisations débutent par un parricide, un infanticide ou un fratricide. «Personne n'a de pire ennemi que son frère, si ce n'est son père qui, craignant une alliance des frères contre lui, prend parfois les devants et les tue, à l'instar de Chronos qui les dévore" (Attali, p. 1216). Si cette "haine vient de la ressemblance», c'est essentiellement dû au fait que «leurs désirs sont les mêmes » créant ainsi rivalité et dangerosité au sein des fratries. Pour maintenir vivante une société, il faut imposer aux « hommes de se méfier de leurs frères. »

Ces « coagulations familiales » (Descola, 2019), qu'elles soient réellement consanguines ou dictées par un intérêt commun pour faire "famille", impliquent un rapport d'autorité et d'adhésion, voire de soumission à cette autorité. Elles se regroupent essentiellement sur un antagonisme intercommunautaire, y compris, dans les récits mythologiques notamment, quand les frères entrent en rivalité. Ces mythes attestent donc les liens complexes qui unissent violence et fraternité.

\section{La verticalité au cœur des fraternités mythologiques}

Pour C. Chalier, les deux sources (grecque et biblique) échafaudent cependant deux conceptions différentes qui irriguent toutes les deux notre civilisation occidentale. Le mythe œdipien et sa déclinaison dans la tragédie antique indiquent que le fratricide réussi serait celui « qui anéantirait le frère avant qu'il ait eu la possibilité d'engendrer une descendance et de lui transmettre son nom » (p. 18), tuant de fait la perpétuation possible des rivaux. Dès lors, Antigone s'entête pour préserver sa fratrie irréconciliable et développe une haine viscérale contre ceux qui l'empêchent d'agir pour sauver ses frères. Ainsi, elle définit la fraternité comme « une relation qui oblige par-delà toute autre considération » (p. 19).

La source biblique lui insuffle, au contraire, une dimension réparatrice pour le couple humain. Comme la tragédie antique, le récit biblique a "une conscience aigüe des menaces de jalousie haineuse qui pèse sur la fraternité mais, contrairement à elle, [il] ne se lasse jamais de relancer l'espoir et d'en chercher les prémices dans la conduite humaine" (p. 24). Cependant, cette vocation rédemptrice ne repose pas sur l'horizontalité d'un projet engageant la relation entre les humains, mais sur la reconnaissance d'une transcendance. "Ce lien de fraternité ne dépend pas d'une décision autonome, prise en toute connaissance de cause ou à ses risques et périls. Il présuppose une orientation par une Parole préalable qui appelle chacun à exister 
comme frère et sœur parmi des frères et sœurs. " (p. 30). Si l'homme doit aimer son prochain comme lui-même, c'est moins pour le reconnaitre d'un seul mouvement comme semblable et autre. Il s'agit de le ramener à la filiation primordiale, en l'englobant dans la communauté que définit par la paternité divine.

Jacques Attali approfondit l'analyse en reliant l'idée de fraternité aux utopies conditionnant la construction ou l'évolution des civilisations. Pour lui, ce sont les codes sociaux qui agissent pour protéger «très soigneusement les frères les uns des autres ». En évitant la victoire d'un frère sur l'autre, la mythologie oriente ou réoriente le destin de la condition humaine. La Bible, ou les textes fondateurs du monothéisme développent largement l'exemple de frères ennemis et de combats entre fratries. Ces épisodes destructeurs conduisent effectivement à l'avènement d'un Messie, porteur d'une utopie incarnée dans l'image d'une éternité plus prometteuse que celle offerte par la condition terrestre. La Fraternité apparait donc dans ces textes, et dans cette perspective messianique, comme :

[...] l'ensemble des conditions permettant à tous les hommes, et donc même aux frères, d'oublier leurs rivalités intrinsèques pour s'aider, s'aimer, se tolérer dans leurs différences et leurs aspirations, prendre du plaisir à la réussite de l'autre, être heureux du bonheur de l'autre. (Attali, p. 1277)

\section{Un but de civilisation?}

Il en conclut que « la fraternité est un but de civilisation, pas un état de nature ${ }^{7}$ (Attali, p. 1215).

Pour C. Chalier, l'apport du texte biblique aux civilisations réside surtout dans la double dimension différenciée et insubstituable de la créature humaine. Ces propriétés permettent de substituer l'idée de "genre humain» à celui de fraternité, «le monothéisme signifi[ant] cette parenté humaine» (Levinas, 1961, p. 236). Ainsi, elle légitime au moins partiellement, l'affirmation de P. Viveret (p. 29) qui considère effectivement que le terme frater désigne essentiellement «le genre humain». Cependant, ce dernier a omis de rappeler l'ancrage biblique du propos, alors qu'il interroge la fraternité républicaine. C'est une confusion très fréquente que nous discuterons.

L'origine des dimensions conceptuelle et axiologique attribuées à la fraternité en occident comme sa déclinaison civilisationnelle sont, on le voit, profondément liées à l'idéologie et aux discours judéo-chrétiens. Cependant, son ancrage républicain plus récent, peut-être issu de la tradition maçonnique comme l'explique Gérard Pithon, tend à vouloir s'en écarter. En effet, la fraternité biblique a vocation à servir l'utopie de l'Éternité initiée par les monothéismes. C'est un fait étymologique qui peut nous conduire à la penser comme " une illusion réservée aux Églises et aux confréries, hors de portée de l'action politique.» (Attali, p. 1289). Qu'en est-il alors de la Fraternité républicaine évoquée par l'institution scolaire notamment?

\section{Laïcité et Fraternité majuscule}

La visée civilisationnelle exposée supra, la permanence de ce concept alors que la République française a séparé les églises et l'état depuis plus d'un siècle, l'essentialité $\mathrm{du}$ principe laïque dans son organisation politique, impliquent d'observer l'impact du politique dans l'évolution du concept. On est alors en droit de se demander si la 
destinée politique ultérieure de la fraternité ne doit pas remettre en cause cette dimension biblique (Chalier, p. 70).

\section{Vers une construction politique}

française, mais là-encore, elle ne s'impose pas comme un fleuve tranquille. Elle charrie, dans ce cadre aussi, violence et désaccord, même si Rousseau la pense au cœur de la fête, comme la liesse d'une foule réunie par le sentiment d'égalité. Selon C. Chalier, pour effacer "hiérarchie et pouvoir", il est nécessaire de "trouver l'expression politique et humaine qui donnera à la fraternité la garantie de la durée en la rendant solidaire de l'égalité et de la liberté.» (p. 73). Il s'agit donc de la penser dans une dimension métapolitique non comme une valeur fédératrice, mais comme un des principes fondateurs de «la chose publique». Elle émane alors d'une construction collective apte à englober les différences interindividuelles au profit d'un commun fédérateur. Pour cela, il faut parvenir à dépasser « le sentiment éphémère et illusoire de la fraternité éprouvé dans la fête » en inscrivant «le principe et la promesse dans la loi » (p. 74) et en l'associant aux principes de liberté et d'égalité. Affirmer ces associations ne suffit pourtant pas à dissocier cet idéal de celui développé à l'aune des monothéismes. L'usage du lien fraternel qui semble se profiler dans les débats révolutionnaires de la fin $\mathrm{du} 18^{\mathrm{ème}}$ siècle l'atteste. Il se revendique essentiellement, comme l'idéal messianique, pour une réunion du genre humain. Il est donc indispensable d'interroger les fondements de cette acception nouvelle pour montrer qu'elle se construit bien dans une véritable disjonction de son origine judéo-chrétienne.

\section{Penser la fraternité au cœur d'un système politique}

Des penseurs comme Bossuet invitaient effectivement à considérer la fraternité humaine à l'image de la paternité divine, permettant ainsi l'Ancien Régime de légitimer un ordre social inégalitaire et injuste (Chalier, p. 75).

Dès 1789 , le concept se renouvèle coupant avec la verticalité d'une transcendance pour s'incarner dans l'horizontalité des principes d'égalité et de liberté aptes à définir une nouvelle organisation politique. De ce fait, il se trouve au centre des utopies révolutionnaires, qui rêvaient d'unir tous les français, puis d'abolir les frontières. Il se pense dans une vocation à l'universalité. Cette promesse, comprise comme un principe fondateur, une vertu, parvient à se frayer « un chemin d'abord sur un plan moral, puis sur un plan juridique » (Ibid.). Elle s'inscrit alors comme une utopie politique pourtant bien vite remise en cause. (Attali, 1999). Effectivement dès 1791, elle réintègre une dimension communautaire, écartant l'ambition d'universalité pour se réduire à une volonté d'uniformisation des individus. Le processus, renouant avec la violence originelle des fratricides, s'incarne alors dans le slogan «la fraternité ou la mort». Ce véritable hymne de ralliement à l'autorité politique décline l'idée de fraternité comme «la forme la plus immédiate et constante de la Terreur » (Sartre, 1960, p. 454). Ce choix 
politique vise à écarter définitivement celui qui se refuse à embrasser l'idéal développé par l'autorité. Il ambitionne de former une société faite d'individus partageant tous les mêmes convictions. Cette fraternité-là, comme celle issue de la Bible, reste fondée sur la foi en un idéal supérieur, qu'il soit religieux ou d'orientation idéologique. Dans tous les cas elle s'incarne dans une autorité politique. Puisqu'elle postule une volonté d'uniformisation de la pensée ou des convictions vers un idéal, elle est nécessairement génératrice d'exclusion d'une partie des autres, ceux qui se refusent à embrasser cet idéal. Il s'agit donc plutôt d'encourager des formes de fraternisation nécessairement éphémères, même si, comme avec l'idéal religieux, elles s'élaborent sur une très grande échelle. Le projet républicain qui se construit progressivement en France de la fin du $18^{\mathrm{e}}$ siècle au début du $20^{\mathrm{e}}$ siècle, cherche, lui aussi, à construire un commun partageable apte à transcender les intérêts particuliers. Cependant, pour y parvenir il se doit de repenser le système politique dans une visée que nous considérons comme génétiquement altruiste. En effet, elle ne dénie pas à l'individu le droit à s'autodéterminer quelle que soit son origine et sa culture primordiale.

\section{Fraternité républicaine et laïcité : un lien consubstantiel}

\section{Une disjonction du mythe originel}

C'est un projet d'une grande ambition que celui de penser une société apte à construire un commun qui englobe le divers sans l'anéantir. Il ne s'agit plus d'agglomérer la somme des individualités mais de penser une société de citoyens capables d'observer autrui comme un semblable malgré ses divergences, parce qu'il est citoyen au même titre que soi. Ainsi conçu, ce rapprochement institue une forme d'" alliance entre des personnes toujours uniques et insubstituables" (Chalier, p.131). La fraternité relève alors essentiellement du politique. Elle s'élabore sur un idéal démocratique, et se disjoint nécessairement de ses origines bibliques et donc de la tradition chrétienne. Le concept se trouve ainsi totalement redéfini. S'il invite les individus à se considérer comme Frères, il ne détermine pas de paternité à cette filiation. Elle doit permettre de penser l'autre comme égal de soi, avec ses dissemblances, donc comme un semblable parce qu'égal dans ses droits et ses libertés.

Pour cette raison, le choix du terme est régulièrement discuté. Aurait-il fallu lui préférer l'idée de "solidarité » pour ne pas entretenir de confusion avec le sens qui lui est conféré par les monothéismes? Faut-il au contraire l'utiliser dans sa dimension laïque, comme le montre Stéphanie Péraud-Puigségur à l'appui de sa "déclinaison législative »? De fait, les deux acceptions sont bien distinctes car "la filiation que suppose [la fraternité politique] et dont elle affirme l'idée ne lui préexiste pas, elle est de nature juridique et historique : les citoyens sont des frères et deviennent du fait qu'ils sont aussi des législateurs, leurs propres fils »(Kintzler, 2014, p. 170).

\section{Un concept nécessairement laïque}

La fraternité républicaine a donc bien vocation à ne pas se prononcer sur la pluralité des fraternités minuscules. L'usage du terme intégrer, utilisé par R. Debray dans la formulation citée en début d'article, est problématique. En effet, il peut laisser penser qu'il s'agirait pour la Fraternité majuscule d'assimiler chacune des fraternités de type communautaire, voire de les reconnaitre. Cependant, le projet vise à garantir à chaque citoyen la liberté de choisir ses appartenances individuelles tant qu'elles ne nuisent pas 
au bien commun. Il est donc essentiel que la République ne se considère pas comme compétente (au sens juridique) au regard des fraternités minuscules. De même, la Fraternité majuscule ne doit pas constituer une entrave à celles-ci, quand elles ne nuisent pas à l'ensemble. Là est contenu tout le sens exigeant et prometteur de cette liberté de conscience que la République s'est engagée à assurer. Pour ce faire, elle a séparé le religieux du politique, en mettant cœur du système politique, constitutionnel et institutionnel le principe de laïcité. Cette acception républicaine de la fraternité noue un lien consubstantiel au principe de laïcité, puisqu'elle ne se reconnait pas dans la verticalité de son essence religieuse ou idéologique. Dès lors, penser un apprentissage de la fraternité au cœur de l'école publique implique de l'engager au regard du principe de laïcité.

Rappelons enfin, à l'instar de C. Kintzler, que le régime laïque ne demande pas aux religions de renoncer "à leur intensité, à leur ferveur, mais à une partie de leur doctrine, celle qui les érige en autorité politique.» (Ibid., p. 34). Cependant, cela ne suffit pas à définir l'exigence politique qu'implique le régime de laïcité, car sans la réciproque le projet perd son sens.

Et on oublie souvent la réciproque de cette coupure : l'autorité politique n'a aucune compétence en matière religieuse, ce qui signifie, bien au-delà de ce qui est admissible en régime sécularisé de toleration, que la reconnaissance morale $d u$ religieux par la puissance publique est impertinente, et aussi que la puissance publique n'a pas le pouvoir d'ériger son propre régime politique et ses prétendues "valeurs" en une religion civile. (Ibid.)

\section{Transmettre des valeurs?}

\section{Les valeurs-principes}

Dans cette perspective, la volonté de transmettre les principes qui fondent la citoyenneté républicaine en les pensant comme des valeurs peut sembler problématique. En effet, « une valeur n'est ni une norme, ni une règle, ni une loi : celles-ci sont des applications de valeurs lesquelles en justifient la création. » (Heinich, 2017, p. 200). La notion de valeur renvoie à l'ensemble des perceptions qu'un individu partage avec son groupe social. La valeur détermine ce qui est licite, souhaitable, acceptable. De fait, elle trouve son pendant négatif : ce qui est illicite, non souhaité, inacceptable. Elle ne peut pas être pensée en dehors de cette articulation des contraires. Les valeurs sont portées par une communauté et déterminent l'agir de ces membres. Dans son analyse visant l'élaboration d'une "sociologie des valeurs», N. Heinich détermine trois acceptions à la "valeur » en fonction du rapport qui s'établit entre le sujet et l'objet dans sa qualification et son processus de valorisation. Avec les notions de valeur-grandeur et de valeur-objet, elle précise le rapport que le sujet entretient à l'objet de valeur ou valorisé. Elle tente alors tour à tour de cerner comment s'exprime le "prix des choses" ou ce que valent les choses mais aussi ce que les individus valorisent, le prix qu'il leur accorde. Puis, elle détermine l'idée de valeurprincipe qui nous intéresse ici. En cela, elle cherche à circonscrire «les différentes catégories de principes d'évaluation et de justification, et leur articulation : autrement dit l'architecture générale qui commandent l'attribution d'une "valeur" [...]» (p. 197). Puisqu'une valeur doit être " à large spectre, temporel autant que spatial : elle implique à la fois le long terme et l'universalité, en tout cas présumée.» (p. 201). Ainsi, ce n'est pas la valeur fraternité en tant qu'objet qu'il faut tenter de transmettre pour former des 
citoyens, mais plutôt les principes qui produisent sa valorisation. Pour ce faire, dans le cas qui nous occupe, il faut se demander «au nom de quoi» (Ibid.) la fraternité républicaine peut être valorisée dans la citoyenneté.

\section{Se penser comme semblable} faut d'abord leur permettre de reconnaitre cette valeur humaine et de comprendre ce qui fonde la citoyenneté elle-même. Pour C. Kintzler, cette construction ne s'élabore pas « par un prêchi-prêcha fusionnel, mais par la constitution d'esprits libres décidés à défendre les droits de tous, y compris de ceux qui ne sont pas "leurs potes" » (p. 174). Il s'agit donc d'apprendre à supporter (aux deux sens du terme) l'autre, même si on le juge insupportable et fort différent de soi ; d'apprendre à construire avec lui cette capacité, car le bien commun le réclame ; finalement d'apprendre à le reconnaitre dans son "humanité ». L'éducation nationale assimile souvent ce projet au nécessaire apprentissage du vivre-ensemble. Là encore, l'expression est trop polysémique pour ne pas être galvaudée. Le vivre-ensemble ne peut être entendu dans le sens communautaire $\mathrm{du}$ terme, mais doit se penser «comme une manière d'apprendre et de progresser ensemble sur fond d'entraide, de coopération, et d'interactions respectueuses des individus, notamment des élèves et des enseignants. » (Jellab, 2019, p. 333). Néanmoins le projet résumé ainsi sonne encore comme un euphémisme. Il s'agit bien par cet apprentissage de préparer les élèves « à vivre en société c'est-à-dire leur donner la possibilité d'une intelligibilité globale du monde qui les entoure, tout en les préparant à y jouer un rôle actif. » (Girault, 1983, p. 75, cité par Jellab, 2019, p. 333). Pour ce faire, nous partageons avec Sylvain Wagnon l'urgence de penser une pédagogie solidaire qui «n'est pas une pédagogie des bons sentiments, [mais] un outil au service d'une action d'envergure pour l'avenir de notre existence. » (2019, p. 23).

\section{Fraternité et éducation}

\section{Penser les valeurs de l'éducation}

Avec ce chapitre, nous entrons dans la visée pragmatique et praxéologique de notre propos. Nous souhaitons ici interroger les conditions requises pour engager une formation aux principes de fraternité favorisant une appropriation de valeurs communes partageables. Cette formation nous apparait comme indispensable pour révéler ${ }^{8}$ notre "humanité ». Bien souvent, l'action dévolue à l'école et les moyens qu'elle devrait mettre en œuvre, se trouvent au centre de polémiques politicomédiatiques, posant des avis jugés fréquemment comme irréconciliables. Cependant, la montée des extrémismes religieux dès la fin du $20^{\text {ème }}$ siècle, la dévalorisation de la pensée critique au profit d'une adhésion rapide et immédiate à des informations non vérifiées, les théories du complot (etc.) incitent l'institution avec une relative 
unanimité, à engager les élèves dans un apprentissage de la citoyenneté et des fondements de ses valeurs. La nécessite de développer une éducation à la fraternité au cœur de l'école fait partie de ces consensus, et trace une visée pour l'école du $21^{\mathrm{e}}$ siècle. Si le but est commun, les moyens pour y parvenir diffèrent.

\section{L'appartenance à l'humanité}

41 Pour Olivier Reboul, les valeurs de l'éducation résident dans «tout ce qui permet à chaque enfant humain de devenir homme» (op. cit., p. 5). La dimension axiologique de notre « appartenance à l'humanité » comprise comme une valeur est déterminée par trois principes qui fondent son universalité : la communication, l'égalité et la solidarité. L'humanité se construit, selon Reboul, sur la possibilité qu'ont les hommes de communiquer et par voie de conséquence d'interagir par-delà les cultures. Elle se détermine dans le postulat d'égalité, pensé comme le droit universel d'être reconnu dans son identité humaine à égale dignité des autres hommes. Elle implique la présence d'une solidarité concrète quand cette dignité est menacée (Ibid., pp. 88-94). Ce sont précisément ces trois principes qui semblent mis à mal par certains aspects de l'évolution de la société.

D'une part, la valeur d'égalité se heurte à la fracture sociale qu'accroit l'absence de régulation du libéralisme économique dans une société globalisée. Elle court alors deux risques à la fois qui, pour Jacques Attali, s'expriment comme des utopies : l'utopie de la liberté et l'utopie de l'égalité dissociée l'une de l'autre. De ce fait, nous considérons avec Jean-Paul Delahaye que la fraternité est «ce qui fait tenir debout notre devise et lui donne tout son sens: pas de liberté ni d'égalité possible entre les citoyens sans fraternité."

D'autre part, la multiplication des échanges distants et fulgurants tant dans leur vitesse de diffusion que dans l'étendue de leur spatialité provoque une déshumanisation de la communication. On sait avec les travaux des anthropologues sur l'invention de l'écriture (Goody, 1979, [1977]) que tout changement dans le mode de communication provoque des bouleversements civilisationnels. Aujourd'hui, la communication n'est effectivement plus hiérarchisée et la connaissance scientifique dévalorisée. Il faut donc permettre aux humains de s'approprier les nouveaux usages langagiers pour maintenir la liberté comme l'égalité par le développement d'une pensée critique. La communication qui préexiste à l'idée de fraternité peut en réguler le sens.

On le voit, aujourd'hui autant qu'hier, même si la hiérarchie des normes se trouve modifiée, l'enseignement ne peut avoir qu'une seule visée globale: émanciper l'individu pour accompagner cette initiation à l'humanité décrite par Reboul.

Alors que faire? Comment faire?

\section{La fraternité au cœur de l'institution}

Les études internationales en font état à chaque publication, le système scolaire français est parmi les plus inégalitaires d'Europe, emporté notamment par l'accroissement des inégalités sociales. Le rapport Delahaye (2015) sur la corrélation entre grande pauvreté et difficulté scolaire témoigne de la nécessité de faire le choix de la solidarité, c'est-à-dire de penser la mixité scolaire comme facteur de justice sociale. Pourtant, certains replis communautaires impliquent des ruptures de solidarité avec 
les exclus, qui, de fait, mettent à mal l'espoir d'une fraternité horizontale et universelle. J.-.P. Delahaye, après avoir exemplifié ce constat, rappelle qu'il est nécessaire de mettre la fraternité au cœur de l'éducation. Pour lui, l'école est au centre de ce combat, et doit avoir pour mission de faire réussir tous les élèves, car l'échec scolaire engendre "des dégâts humains et un immense gâchis ». Une telle affirmation en conclusion de son propos interroge : que signifie « faire réussir » tous les élèves?

Tout d'abord, le système scolaire doit pouvoir assurer une qualification professionnelle à chacun: ce défaut de qualification reste un des piliers de la relégation sociale. Aujourd'hui, ce point semble faire l'objet d'un consensus national. Par voie de conséquence, les statistiques témoignent d'une évolution positive perceptible dans cette dernière décennie.

Cependant, la réussite se réalise aussi quand l'individu peut développer les potentialités qui lui permettront de s'ouvrir aux différents savoirs et savoir-faire, à la culture, c'està-dire à autre chose qu'à lui-même (Reboul, p. 94). L'école doit donc être à même de favoriser l'émancipation intellectuelle et sociale de l'élève pour lui permettre de trouver sa place et de développer ses talents individuels. Les pratiques pédagogiques mises en œuvre au cœur d'une école fraternelle doivent de ce fait élaborer des "finalités de justice sociale et de liberté »(Wagnon, 2019, p.40). Gérard Pithon, présentant le projet pédagogique d'un Établissement Régional d'Enseignement Adapté (EREA) apporte une pierre à cette réflexion par la construction d'une typologie des attentes des adultes mais aussi des élèves : bienveillance, professionnalisme, équité et humour. Bien loin de l'image d'une réussite scolaire passant par des filières d'excellence exclusivement définies préalablement par l'élite, cette recherche exploratoire témoigne du besoin d'humanité exprimé par les élèves qui ont participé à ce programme. Pour ces élèves, le professionnalisme de l'enseignant réside dans la capacité à « bien aider pour les devoirs, mieux expliquer, rendre service, s'intéresser à [leur] travail ». Ces enfants, qui ont tous rencontré une difficulté scolaire importante, confirment que l'éducation ne peut être dissociée de l'instruction, que l'accès aux savoirs ne se construit pas uniquement dans la verticalité d'une relation entre un magister de la connaissance (maitre) et son disciple l'élève. Ainsi, ils donnent « la priorité à la formation et non à la seule transmission des connaissances» (Wagnon, Ibid.). L'apprentissage de la citoyenneté à l'école passe par la création d'un lien de solidarité au cœur de la classe, qui s'exprime dans la pédagogie mise en œuvre.

Dans cette perspective, il faut savoir prendre en compte les mutations et les besoins de notre société pour penser les manières d'enseigner. Nous l'avons dit, le projet d'une école soucieuse de penser la citoyenneté vise à construire « des esprits libres décidés à défendre les droits de tous» (Kintzler, p. 174). Force est alors de constater que «la transmission frontale ne correspond en rien à une réflexion libre et ouverte, que la mémorisation ne peut être l'apha et l'oméga de [ces] apprentissages » (Wagnon, p. 53). C'est effectivement sur ce double postulat créer des esprits libres et forger des citoyens solidaires que se construisent chacune des propositions scientifiques du dossier.

\section{Quelques propositions et réflexions pédagogiques}

\section{Remarques liminaires}

Bien sûr, cette contribution n'a pas prétention à apporter ensemble une vision exhaustive. Elle développe quelques propositions concrètes pour une construction 
kaléidoscopique d'une pédagogie solidaire parce qu'elle reste en permanence soucieuse de la construction intellectuelle et du développement des aptitudes de chacun.

Nous ne reviendrons pas sur l'importance capitale de l'enseignement de l'histoire, auquel Aziz Jellab, notamment, consacre un chapitre fort éclairant dans son ouvrage (op. cit, p. 325-334). Nous considérons aussi, à l'instar de Bruno David que « la question $\mathrm{du}$ « fait » [...] est le fondement de toute discipline scientifique.» (Proust \& Lecointre, 2019, p. 3). Son dévoiement permanent, tant sur le plan sémantique que dans la remise en question des méthodes qui permettent de l'élaborer, mettent à mal le développement d'une pensée rationnelle.

51 Nous nous sommes concentrés sur la double problématique des langages et de la communication: elle traverse l'ensemble des articles réunis dans le dossier interrogeant quelques-unes des formes que peut prendre une éducation à la fraternité. Nous avons aussi souhaité que chaque article analyse la manière dont les dispositifs didactiques ou pédagogiques, en construction ou réalisés, puissent contribuer à favoriser une appropriation du concept de fraternité par les élèves, les incitant alors peut-être à savoir prendre un jour la décision de fraternité.

\section{Apprendre par et avec l'écriture}

Pour Yves Soulé, cet apprentissage débute dès les prémisses de la scolarisation, à l'école maternelle, quand des élèves non-lettrés s'entrainent à écrire. Sa contribution « envisage donc la fraternité là où elle n'est guère questionnée et où elle permettrait peut-être une mise en œuvre plus efficace: dans les situations d'enseignement/ apprentissage ordinaires, loin des activités réactives, des projets "spectaculaires", des manifestations médiatisées.» Bien qu'ordinaire, la situation choisie qui vise les "premiers apprentissages de l'écriture à l'école maternelle » n'en est pas moins essentielle à la formation du jeune élève. L'écriture, qui nécessite "une implication individuelle » interroge avant tout le rapport à la communication, tant dans la mise en œuvre de l'apprentissage que dans sa finalité. Ainsi, elle se situe bien au cœur des principes soutenant la valeur «d'appartenance à l'humanité » évoquée précédemment à l'instar des travaux d'O. Reboul. Certes, l'apprentissage du lire-écrire en lui-même est émancipateur. Cependant, pour le chercheur, cette situation ne suffit pas à développer le rapport de solidarité. C'est dans la puissance du dispositif didactique construit et réalisé, qui implique de savoir articuler les constantes verticales des formes de modélisation et les singularités horizontales de la différenciation, que s'élabore progressivement la coopération nécessaire à la prise en compte de l'autre. Apprendre ensemble à écrire pour soi comme pour l'autre.

Pour S. Bazile, P. Dequin, D. Heches, la communication écrite est aussi au cœur de la formation à la fraternité. S'appuyant sur l'analyse d'une mise en œuvre du projet Eduquer à l'égalité dans la diversité, proposé par la Ligue de l'enseignement, les autrices interrogent la pratique d'une enseignante dans un CP-CE1 d'une école perpignanaise qui veut élaborer " une fraternité en actes et en paroles ». Faisant surgir l'inconnu au cœur de la classe par la présentation d'un jeu de six photographies représentant des formes d'altérité, le dispositif didactique élaboré par la professeure invite à construire oralement une "définition kaléidoscopique de la fraternité ». À l'issue de cette première phase du travail, les élèves doivent choisir une photographie utilisée comme une carte. Puis, ils rédigeront un message de fraternité destiné à un «étranger » qui pourra répondre poste restante. L'analyse des travaux et d'un corpus d'entretiens 
conduits une fois achevé ce travail plutôt surprenant interroge précisément la pertinence d'une telle action ponctuelle pour engager l'élève dans une appropriation du concept républicain.

\section{La fiction littéraire comme expérience de l'altérité}

Les articles proposés par A. Perrin-Doucey et J. Perrin, ou par A. Gennaï considèrent le rôle de la fiction littéraire dans le développement d'une nécessaire pensée critique. Ainsi, ils apportent une contribution supplémentaire à la réflexion épistémologique et didactique engagée depuis quelques années sur la place de la littérature dans l'enseignement des valeurs.

Pour A. Perrin-Doucey et J. Perrin, c'est sur la lecture littéraire, pensée comme va et vient dialectique entre émotion et raison que se centre l'étude. S'appuyant sur deux extraits de La Fortune des Rougon, les auteurs se demandent comment la fiction littéraire est à même "d'apprendre à accueillir l'autre dans sa dissemblance ". L'analyse diégétique des extraits permet de repérer les ressources axiologiques mobilisées par ces textes, notamment dans l'agir des personnages. C'est un levier pour anticiper la construction de dispositifs didactiques offrant aux lecteurs la possibilité d'interroger par l'expérience fictionnelle «les conséquences politiques d'un certain oubli de la fraternité dans les sociétés modernes. » (Bidar, p. 94).

A. Gennaï, quant à lui, se demande dans quelle mesure la confrontation au texte patrimonial peut favoriser la reconnaissance par l'élève d'une culture commune partageable. Il montre tout d'abord, à l'appui des travaux de Brigitte Louichon, que l'œuvre patrimoniale et ses adaptations progressives peuvent contribuer à établir ou repenser un lien intergénérationnel. Il interroge ensuite, à partir de l'étude de la Belle et la Bête en classe de cycle 3, de façon prospective, la manière dont « l'objet patrimonial, le processus de patrimonialisation et les modalités d'enseignement peuvent contribuer à une éducation à la fraternité ».

\section{Philosophie et développement de la pensée critique}

C. Budex réaffirme l'impact crucial sur la vie sociale et philosophique de cet apprentissage, qu'il considère à juste raison comme un impensé de l'école. Il rappelle d'une part le cadre laïque de l'enseignement du concept de fraternité à l'école, d'autre part la place de l'enseignement moral et civique dans la formation intellectuelle des élèves. Puis, il interroge la contribution que la didactique de la philosophie peut apporter à ce projet. Pour ce faire, il analyse des pratiques effectives de Discussion à Visée Philosophique (DVP) recueillies dans le cadre de sa recherche doctorale. Il veut montrer que cette dernière permet «dans sa forme, [de] développer des dispositions éthiques « fraternelles » en tant que dispositif de dialogue philosophique, [et qu'] elle le peut également en interrogeant, dans son contenu, la notion de fraternité et ses enjeux. »

58 S. Péraud-Puigségur revient, quant à elle, sur les travaux de la philosophe Martha Nussbaum (2010/2011) sur les « émotions démocratiques » qu'elle interroge à l'aune de la problématique de la fraternité. Elle met alors en évidence trois problèmes inhérents à ce concept développé par la lecture des travaux philosophiques de Nussbaum: «la nature même de la fraternité » comprise dans sa verticalité étymologique, les « enjeux d'une éducation à la fraternité » et les «moyens de réalisation de cette éducation à la 
fraternité et de la formation de la sensibilité ». Elle cherche ainsi à comprendre «en quoi la pensée de Martha Nussbaum permet de les approfondir et de les clarifier, sinon de les résoudre ».

\section{Penser l'organisation scolaire}

La contribution de G. Pithon, qui clôt cet ouvrage, nous offre aussi l'occasion de clore notre introduction. Il revient, à l'instar d'Y. Soulé sur les gestes professionnels pédagogiques mis en œuvre dans la formation des élèves. En effet, l'organisation pédagogique en tant que telle, conçue pour mettre en actes les valeurs inhérentes à la fraternité républicaine, forme le cœur du dispositif analysé. G. Pithon se demande comment les relations enseignants-élèves et les dispositifs d'accompagnement personnalisé peuvent concourir à la construction citoyenne. Pour ce chercheur, l'histoire de la devise, sur laquelle il revient avec précision, met en évidence l'impact du politique dans l'institution des rapports maitre-élève au sein de l'école. Il postule alors que dans une république qui conçoit sa devise autour des valeurs de liberté, d'égalité et de fraternité, il est essentiel pour favoriser leur apprentissage, de prendre en compte les compétences psycho-émotionnelles des élèves pour penser des parcours individualisés. Il s'appuie ensuite sur les données d'une recherche exploratoire conduite dans un Établissement Régional d'Enseignement Adapté montpelliérain pour décrire ce choix pédagogique. Il interroge la constitution et les effets du dispositif éducatif (le contrat pédagogique personnalisé) conçu pour «tenter d'améliorer le climat "fraternel" dans une "communauté éducative" " pour montrer qu'il facilite une remise en cause personnelle, favorisant ainsi la prise en compte de l'altérité.

Avec un tel dispositif, la décision de fraternité n'a plus à être prise, elle s'institue au cœur même de l'organisation pédagogique qui préside au développement des compétences et à l'appropriation des savoirs par les élèves.

\section{BIBLIOGRAPHY}

Attali, J. (1999). Fraternités : Une nouvelle utopie. Paris : Fayard.

Bidar, A. (2015). Plaidoyer pour la fraternité. Paris : Albin Michel.

Chalier, C. (2003). La fraternité un espoir en clair-obscur. Paris : Buchet/Chastel. Coll. au fait.

Debray, R. (2009). Le moment fraternité. Paris: Gallimard. Coll. Folio essai.

Descola, P. (2019). Présentation du séminaire individuation, individualité, individualisme. Collège de

France, 31 janvier 2019. https://www.college-de-france.fr/site/philippe-descola/

seminar-2019-01-31-10h00.htm

Gaffiot, F. (1934). Dictionnaire illustré latin-français. Paris : Hachette.

Girault, R. (1983). L'histoire et la géographie en question, Rapport au ministre de l'éducation nationale, 1983. Paris : Ministère de l'éducation nationale. 
Goody, J. (1979, [1977]). La Raison graphique. La domestication de la pensée sauvage, traduit de l'anglais par J. Bazin et A. Bensa. Paris : Éd. de Minuit.

Heinich, N. (2017). Sociologie des valeurs. Paris : Gallimard. Coll. NRF.

Jellab, A. (2019). Une fraternité à construire. Essai sur le vivre-ensemble dans la société contemporaine. Boulogne-Billancourt : Berger Levrault.

Kintzler, C. (2014). Penser la laïcité. Paris : Minerve.

Levinas, E. (1961). Totalité et infini. La Haye : Éd. Nijhoff.

MEN. (2015a). Onze mesures pour une grande mobilisation de l'École pour les valeurs de la République. http://www.education.gouv.fr/cid85644/onze-mesures-pour-une-grande-mobilisation-de-lecole-pour-les-valeurs-de-la-republique.html

MEN. (2015b). L'enseignement moral et civique (EMC). Bulletin officiel spécial du 25 juin 2015. https://www.education.gouv.fr/cid90776/l-enseignement-moral-et-civique-au-bo-special-du-25juin-2015.html

Morin, E. (2019). La Fraternité, pourquoi? Arles : Actes Sud Nature.

Proust, S. Lecointre, G. (dir.). (2019). Le fait en question. La Tour d'Aigues : Éditions de l'Aube.

Sartre, J.-P. (1960). Critique de la raison dialectique. Paris : Gallimard.

Reboul, O. (1992). Les valeurs d'éducation. Paris : Presses Universitaires de France.

Vallaud-Belkacem, N. (2015). Présentation de la grande mobilisation pour l'École pour les valeurs de la République. 22 janvier 2015. https://www.education.gouv.fr/cid85665/presentation-grandemobilisation-pour-ecole-pour-les-valeurs-republique.html.

Viveret, P. (2015). Fraternité, j'écris ton nom! Paris : Les liens qui libèrent.

Wagnon, S. (2019). Pour une pédagogie solidaire. Aniche : Siphayat.

\section{NOTES}

1. Dorénavant MEN.

2. Nous évoquons ici les incidents qui ont émaillé au sein des écoles les temps dédiés aux minutes de silence en hommage aux victimes de cette série d'attentats : refus de participer, prises de position exprimant une forme d'adhésion passive aux actes terroristes, remise en question de la réalité de ses attentats, théorie du complot, etc.

3. Colloque interuniversitaire Fraternité en éducation, éducation à la fraternité un enjeu pour l'école du $21^{1 e ̀ m e}$ siècle. Université de Montpellier, Faculté d'éducation, 24 et 25 mai 2016.

4. Nous empruntons ce néologisme à Edgar Morin, propos tenu dans son séminaire Le défi des complexités problèmes et méthodes. Séance 1. Université de Montpellier, Faculté d'éducation. 16 janvier 2019. https://video.umontpellier.fr/video/2443-seminaire-edgar-morin-14-le-defi-descomplexites-problemes-et-methodes/

5. Parfois nommée comme une mêmeté.

6. Ici, selon J. Attali (1998), la question est avant tout pensée dans une composante masculine, la sororité impliquant d'autres mouvements qui se déclinent différemment dans les récits mythologiques.

7. En italique dans le texte

8. Le verbe révéler doit être compris selon l'usage que lui donne l'expérience du tirage d'une photographie argentique. 


\section{AUTHOR}

\section{AGNĖS PERRIN-DOUCEY}

Université de Montpellier, LIRDEF 